Revue de l'Institut des langues et cultures

d'Europe, Amérique, Afrique, Asie et Australie

$28 \mid 2017$

Passages, ancrage dans la littérature de voyage

\title{
Le voyage et les usages de l'espace méditerranéen à l'époque du Grand Tour
}

Travelling in the Mediterranean: Uses and Practices at the Time of the Grand

Tour

\section{Gilles Bertrand}

\section{OpenEdition}

Journals

Édition électronique

URL : http://journals.openedition.org/ilcea/4087

DOI : 10.4000/ilcea.4087

ISSN : 2101-0609

Éditeur

UGA Éditions/Université Grenoble Alpes

Édition imprimée

ISBN : 978-2-84310-374-2

ISSN : 1639-6073

Référence électronique

Gilles Bertrand, «Le voyage et les usages de l'espace méditerranéen à l'époque du Grand Tour », ILCEA

[En ligne], 28 | 2017, mis en ligne le 06 mars 2017, consulté le 30 avril 2019. URL : http://

journals.openedition.org/ilcea/4087 ; DOI : 10.4000/ilcea.4087

Ce document a été généré automatiquement le 30 avril 2019

(c) ILCEA 


\title{
Le voyage et les usages de l'espace méditerranéen à l'époque du Grand Tour
}

\author{
Travelling in the Mediterranean: Uses and Practices at the Time of the Grand
}

Tour

Gilles Bertrand

1 Si la Méditerranée est au sens premier une mer qui a depuis longtemps été sillonnée par les peuples riverains, le terme englobe aussi à l'image du Mare Nostrum des Romains un ensemble de pays qui la bordent et il désigne de ce fait tout à la fois des zones maritimes et terrestres (Pelletier \& Bergès, 2008). C'est d'ailleurs bien le sens que lui donnait Fernand Braudel dans sa fameuse thèse sur la Méditerranée à l'époque de Philippe II (Braudel, 1949). On peut cependant se demander comment les rituels du voyage d'agrément et de connaissance du Grand Tour, nés au milieu du XvI siècle en Angleterre, l'ont impliquée. Le Sud de la France et la péninsule italienne jusqu'à Naples furent les seuls points de la Méditerranée atteints par les itinéraires surtout continentaux qui se déployèrent pour favoriser l'insertion des jeunes aristocrates européens dans l'univers des cours et les aider à acquérir un goût poli par le contact avec les œuvres d'art. Mais le Grand Tour n'épuise pas la multiplicité des voyages accomplis par les élites du vieux continent, et a fortiori il peine à intégrer ceux qui s'effectuèrent en Méditerranée. En revanche, il s'inscrit dans une stratégie du voyage d'étude qui a caractérisé l'époque

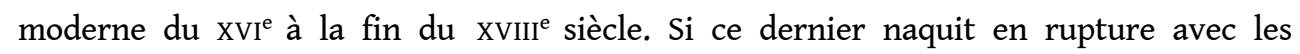
pèlerinages vers Jérusalem, il coexista pour partie à leur vogue, puis finit par y substituer des pratiques laïcisées. Malgré un fléchissement dans les premières décennies du XvI siècle, les récits de pèlerins connurent encore une embellie au début du XvII ${ }^{\mathrm{e}}$ siècle, alors que déjà le Grand Tour s'affirmait. De manière générale c'est dans ce contexte qu'à côté de la course, du cabotage, du commerce des captifs et des expéditions militaires, on vit se développer à partir du $\mathrm{XV}^{\mathrm{e}}$ siècle en Europe comme en Méditerranée des modes de déplacement physique qui, dans le cas des pèlerins en quête de leur salut autant que dans celui des jeunes gens désireux d'apprendre, débouchèrent sur une floraison d'écrits 
intimes, littéraires ou scientifiques, de descriptions et de guides (Pouillon, 2008). Ces écritures produisirent une conscience du voyage et le firent aller bien au-delà d'une simple activité utile ou contrainte.

2 Sous des formes variées, ces textes ont contribué à créer un espace imaginaire (Tinguely, 2000). Celui-ci fut certes marqué par des projets religieux, des conflictualités politiques et des peurs tenaces, mais il fut également de plus en plus modelé au cours de l'époque moderne par la curiosité savante et le besoin du divertissement cultivé. Espace charnière entre l'Orient et l'Occident, la Méditerranée traversée par les voyageurs de Venise à la Grèce et à l'Empire ottoman, du Levant à l'Égypte et au Maghreb, Maroc compris, et même jusqu'à l'Espagne, relevait-elle de l'Orient des Européens, de l'Occident des Orientaux, ou bien constituait-elle un univers autonome ? Elle semble être restée jusqu'au seuil du XIX siècle un monde intermédiaire et éclaté, par où l'on passait plus que l'on ne cherchait à y séjourner. Son identité aurait été tirée de son nom, soulignant sa position « au milieu de toutes les terres de l'ancien Monde ", qu'elle divisait « en trois parties, qui sont l'Europe, l'Asie \& l'Afrique $»^{1}$. Un désir de l'espace maritime, de ses îles et de ses côtes ne s'y exprime que peu à peu. La multiplicité des voyages qui s'y déployèrent du nord-ouest vers l'est et le sud-est mais aussi du sud vers le nord, contribua néanmoins à créer une fascination. Face aux musulmans qui gagnaient l'Europe, un nombre croissant d'Européens cheminant vers l'Orient y vit à la fois le lieu de la séparation et celui du retour possible de chacun à ses origines.

\section{Un espace de circulation traditionnel mais risqué}

Bien que familier, le voyage en Méditerranée demeura à l'époque moderne une épreuve redoutable. Si Jean Delumeau a davantage inventorié les peurs que suscitaient en Occident les voyages transocéaniques ou ceux effectués dans les mers du Nord que ceux en Méditerranée (Delumeau, 1978: 31-42), Alain Corbin a montré comment il fallut attendre plus longtemps qu'en Europe septentrionale, dont les littoraux furent apprivoisées dès le milieu du XVIII ${ }^{\mathrm{e}}$ siècle, pour que les rivages du Sud cessent d'apparaître hostiles (Corbin, 1988 : 171-186). Les risques inhérents aux navigations méditerranéennes s'inscrivaient à vrai dire dans une longue durée: pirates barbaresques, corsaires, flibustiers et autres "tyrans de la mer " (Requemora \& Linon-Chipon, 2002), tempêtes provoquant des naufrages à l'instar de celui de Jean Palerne en 1581 au large de Rovigno en Istrie tandis qu'il se rendait à Alexandrie d'Égypte ${ }^{2}$, mal de mer subi par le président de Brosses en 1739 entre Menton et Vintimille sur le chemin de l'Italie ${ }^{3}$. Ces périls contribuèrent à renforcer des stéréotypes et prolongèrent des psychoses. Un lucratif marché des "captifs" amenait des musulmans dans les ports de Gênes, Livourne, Messine, Trieste et surtout Malte avec un faible espoir de rachat par les souverains musulmans, tandis que des chrétiens faits prisonniers par les pirates étaient eux-mêmes soit vendus et réduits en esclavage sur les marchés d'Afrique du Nord (Davis, 2006), soit rachetés (Kaiser, 2008; Moureau, 2008) ou, dans le meilleur des cas, abandonnés sur les côtes de Corse à l'instar du lieutenant de cavalerie Jean Coppin, au retour de son voyage en Egypte en $1638^{4}$.

4 Les dangers naturels se combinaient avec les impératifs de la politique, provoquant l'échec d'expéditions comme celle de Charles Quint contre Alger en 1541. Largement oubliée, celle-ci manifesta une volonté de maîtriser la Méditerranée qui se heurta, à la différence de l'expédition de 1535 à Tunis, autant à la tempête qu'à la démesure du projet 
et à la résistance des Algérois (Nordman, 2011). Le regard sur l'autre musulman porté par l'Europe chrétienne subit durablement l'impact de la perception d'une menace turque après la prise de Constantinople en 1453. La France tenta de construire à partir de François $\mathrm{I}^{\mathrm{er}}$ un rapport d'alliance privilégié avec le sultan. Catholiques et protestants se disputèrent des zones d'influence en Méditerranée orientale comme le montre La Syrie sainte du jésuite Joseph Besson (1660), et la composante religieuse irrigua dans la seconde moitié du XVII e siècle les projets de nouvelle croisade contre l'Empire ottoman. Théâtre de conflictualités durables entre ce dernier et l'Occident, lesquelles avaient amené à assimiler au XVI ${ }^{e}$ siècle le mot voyage à une « campagne de guerre ${ }^{5}$ » et culminé dans la bataille de Lépante, où la flotte chrétienne était parvenue à mettre en échec la marine ottomane (1571), la Méditerranée fut aussi un espace de l'impasse militaire entre puissances européennes, lorsque les Anglais détruisirent à Aboukir en 1798 la flotte de l'expédition d'Égypte dirigée par Napoléon Bonaparte. Les guerres suscitèrent même des détournements par voie de terre. Soucieux d'éviter les effets du blocus continental, Napoléon imagina de mettre au point un réseau de canaux en Italie du nord et centrale pour faire transiter les troupes et les marchandises de la France vers la mer Adriatique et les Balkans sans passer par la Méditerranée.

5 Certes la Sicile avait été entre le $\mathrm{x}^{\mathrm{e}}$ et le $\mathrm{XII}^{\mathrm{e}}$ siècle visitée par de grands voyageurs ou géographes arabes venus du Kurdistan comme Ibn Hawqal ou de l'Espagne comme Al Idrissi et Ibn Jubayr (Ruta, 2009). Les voyages en Méditerranée furent cependant à l'époque moderne un peu moins ceux des Turcs ou Arabes vers l'Europe chrétienne que des Européens vers le Levant ou le Maghreb, alors appelé Barbarie et dominé par les régences d'Alger, Tunis et Tripoli. Sauf exception, attestée par la présence de trente mille Turcs en 1543 à Toulon, les musulmans n'atteignirent les terres jouxtant de près ou de loin la Méditerranée occidentale, et notamment la France, que de façon sporadique avant le XIX siècle (Dakhlia \& Vincent, 2011; Valensi, 2012). À côté des envoyés ottomans comme Süleyman Agha, dépêché en 1669 par le sultan auprès de Louis XIV, ou de ceux des régences barbaresques gagnant depuis le XVI ${ }^{\mathrm{e}}$ siècle la cour de France, on relève en France cinq catégories de voyageurs orientaux à l'époque moderne (Poumarède, 2006). C'étaient des domestiques ou secrétaires ramenés par le personnel des ambassades et consulats, quelques marchands grecs, juifs, arméniens, plus rarement turcs, égyptiens, tunisiens et algériens, ou bien encore des soldats comme les Tartares et Turcs du régiment de SaxeVolontaire entre 1743 et $1750^{6}$. Parfois des musulmans se déplaçaient pour faire valoir leurs droits, tels ces Turcs de Crète arrivés à Marseille en 1728 pour obtenir justice d'un acheteur qui ne leur avait pas payé une cargaison d'huile. Enfin, de nombreux captifs étaient saisis dans les navires barbaresques ou achetés sur les marchés d'esclaves pour être engagés comme rameurs.

6 Venus à l'inverse d'Europe, les pèlerins qui allaient en Terre Sainte parcouraient la BasseÉgypte et le Sinaï et donnèrent de l'espace méditerranéen une image dans laquelle la chrétienté s'est réfléchie. La Peregrinatio in terra sanctam du chanoine de Mayence Bernhardt von Breydenbach, publiée en latin et en allemand en 1486 avec une carte de Palestine et quatre grandes vues de villes, fut suivie de nombreuses relations comme celles, au XvI ${ }^{\mathrm{e}}$ siècle, du marchand de draps Jacques Le Saige, du prêtre Denis Possot ou du seigneur Jacques de Villamont (Gomez-Géraud, 1999). L'arrivée se faisait de l'ouest par l'Espagne, Marseille, Gênes, Venise, Ancône ou Brindisi, et du nord ou de l'est par Pula, Raguse ou la mer Noire. Prolongé par ses " annexes », la mer Adriatique, la mer Égée ou les Détroits, le Mare Nostrum offrait ses îles à la curiosité de ces religieux, nobles ou 
marchands: la Sicile, la Sardaigne, Corfou, la Crète, Rhodes, Chypre, les «îles de l'Archipel » et bien d'autres. Malgré leur attrait pour les Turcs, les femmes voilées, la liberté des chrétiens d'Alep ou la sûreté des rues du Caire, les pèlerins se sentaient néanmoins mal à l'aise face à la bigarrure ethnique et à la coexistence religieuse dans les villes et les ports de la Méditerranée (Bonnin, 2001).

Les missions diplomatiques induisaient un regard plus empathique, dont témoignent les relations à Constantinople et dans le Levant de Jean Chesneau (1547-1555), Philippe Canaye du Fresne (1573), François Savary de Brèves (1584 à 1605) ou Henry de Beauvau (1604-1605). Ce dernier, dans sa Relation journalière du voyage du Levant parue en 1608, exprime de manière explicite à la fois le désir de servir son roi et son inclination à la curiosité traduite dans les vignettes gravées qui accompagnent son livre. La même attitude se dégage des relations de Louis Deshayes de Courmenin (1621), du sieur Du Loir parti de Marseille en 1639 avec l'ambassadeur du roi de France avant de revenir l'année suivante avec celui de Venise, du père Robert de Dreux, aumônier d'ambassadeur (1665-1669), enfin des Mémoires du chevalier Laurent d'Arvieux, qui de 1653 à 1686 ajouta à ses activités commerçantes et consulaires celles de négociateur à Tunis, Istanbul et Alger. Les missions diplomatiques anglaises ne furent pas moins nombreuses, qui notamment sous les règnes de Jacques $\mathrm{I}^{\mathrm{er}}$ et Charles $\mathrm{I}^{\mathrm{er}}$ occasionnèrent des voyages vers le Levant, Constantinople ou l'Égypte ${ }^{7}$. Ayant contribué à la fin des années 1770 à réorganiser les Échelles du Levant et de Barbarie et préparé une expédition française en Égypte annonçant celle de Bonaparte, le baron François de Tott publia en 1784 des Mémoires sur les Turcs et les Tartares. Fort de ses longs séjours de Constantinople à la Crimée et à l'Égypte entre 1755 et 1779, il y décrit le despotisme ottoman au quotidien (Shahadeh, 2016).

\section{Le voyage comme entreprise de collecte de connaissances}

Combinant la crainte en même temps que la curiosité pour l'inconnu, les voyageurs qui pratiquaient la Méditerranée voulurent l'apprivoiser. Initiée dès la fin du XIII siècle, la science cartographique culmina avec les travaux de Joseph-Bernard Chabert dans les années 1753-1759, avant de déboucher en 1830 sur un Neptune de la Méditerranée. Cette compilation réunissait les cartes de côtes réalisées depuis l'époque où Colbert avait envoyé une mission annuelle d'ingénieurs le long des rivages de la Méditerranée entre 1679 et 1688. Les hydrographes se dépensèrent sans compter pendant des siècles pour parfaire la connaissance des côtes et établir des cartes utiles aux navigateurs et, partant, aux voyageurs. Ainsi du Portulan de la mer Méditerranée, ou Le vray guide des pilotes costiers, publié par le navigateur Henry Michelot en 1703 avec le souci de décrire tous les ports, havres et abris et de montrer « la véritable maniere de Naviguer le long des Côtes d'Espagne, Catalogne, Provence, Italie, les Isles d'Yvice, Mayorque, Minorque, Corse, Sicile, \& autres $»^{8}$.

9 Liée au déploiement de l'activité politique et diplomatique, la volonté de connaissance de l'espace physique se traduisit dès le XVI $I^{e}$ siècle par le voyage au Levant et en Égypte de Pierre Belon du Mans (1546-1549). Protégé d'un ambassadeur auprès du Grand Turc, cet apothicaire français étudia la flore, en particulier les simples, la faune, les minéraux et autres curiosités pittoresques 9 . De leur côté quelques Britanniques firent de la 
Méditerranée au début du XVII ${ }^{\mathrm{e}}$ siècle un laboratoire de savoirs naturalistes, historiques et ethnologiques : William Lithgow se rendit à pied entre 1609 et 1629 des Shetland à la Crète en passant par la Suisse et la Bohème et il marcha de la Palestine jusqu'à Fez avant d'être arrêté comme espion à Malaga, tandis que le poète et futur colon en Amérique du Nord George Sandys partait en 1610 pour Constantinople puis l'Égypte, le Mont Sinaï et la Palestine avant de gagner Chypre et l'Italie, tirant de son voyage une relation dédiée au futur roi Charles $\mathrm{I}^{\mathrm{er}}$ qui fait autorité en matière de géographie et d'ethnologie ${ }^{10}$. Plus tard, Joseph Pitton de Tournefort rapporta huit mille plantes de son voyage en Orient (1700-1702) et Déodat de Dolomieu affina dans les îles Éoliennes en 1781 sa connaissance du volcanisme avant d'effectuer chaque été des explorations dans les Alpes. Les missions savantes se multiplièrent dans les dernières années du siècle. Celle de l'entomologue Guillaume-Antoine Olivier et du spécialiste de coquillages Jean-Guillaume Bruguière, envoyés par le gouvernement français en 1792 dans l'Empire ottoman, en Égypte et en Perse, déboucha sur une relation éclectique publiée à partir de 1800 par Olivier ${ }^{11}$. Elle révèle les difficultés d'une entreprise vouée à la connaissance : aléas des vents, animosité des habitants de Santorin, attaque des Kurdes, foudres du pouvoir impérial pour avoir dressé le portrait impitoyable de certains pachas, maladie de Bruguière qui mourut sur le chemin du retour, entraînant un conflit dû à une appropriation des travaux du défunt par son compagnon.

10 Non sans être marqués par les enjeux politiques ou religieux, les commerçants, gens de lettres, artistes, médecins et autres savants rendirent également compte à partir du Xvi siècle des mœurs et coutumes des peuples de la Méditerranée. Le récit de Jean Palerne, contemporain de Montaigne, qui voyagea pour son plaisir en Grèce, Turquie, Syrie, Palestine et Égypte (1581-1583), abonde en remarques alertes sur les comportements et pratiques religieuses des musulmans ${ }^{12}$. Mus par un esprit d'aventure, Nicolas de Nicolay (1551) ou Jacques de Villamont (1588-1590) embrassèrent dans leurs voyages la géographie physique et humaine, l'ethnographie, la politique, l'économie, l'histoire, la religion et même la navigation, expérimentant la diversité des embarcations, des vents, des ports et des arsenaux ${ }^{13}$. Un immense succès fut remporté dans l'Europe du XVII ${ }^{e}$ et du $\mathrm{XVIII}^{\mathrm{e}}$ siècle par les relations des six voyages vers l'Orient du négociant en pierres précieuses Jean-Baptiste Tavernier, accomplis de 1630 à 1668, ainsi que par les notations de Jean Thévenot qui circula entre 1655 et 1659 de la Turquie à Tunis ${ }^{14}$. Le fils d'orfèvre Paul Lucas, marchand et antiquaire, fit paraître les siennes à l'issue de quatre voyages effectués de 1688 à 1717 au Levant, en Grèce, en Asie mineure et en Égypte ${ }^{15}$. Chacun d'entre eux raconta la Méditerranée, parfois sur la route de la Perse et des Indes.

11 Sans retirer au Père Pierre Dan la primeur de son Histoire de Barbarie et de ses corsaires (1637), ni à l'Anglais Thomas Shaw le mérite d'avoir fait de ses voyages en Barbarie et au Levant vers 1732 une référence incontournable pour le XVIII ${ }^{\mathrm{e}}$ siècle, des voyageurs français entre autres spécialistes de la flore visitèrent les régences d'Afrique du Nord : ce fut le cas de Jean-André Peyssonnel (1724-1725), René Desfontaines (1783-1785) et l'abbé Jean-Louis Marie Poiret (1785-1786). Partageant les idées de l'époque sur l'immobilisme de ces États formellement soumis à l'autorité du sultan, ils ne perçurent pas que la Régence d'Alger glissait alors d'un pouvoir absolu mais anarchique exercé par le représentant du sultan vers un système oligarchique plus stable, ce que les rapports des consuls en revanche mentionnaient (Brahimi, 1976). 


\section{Réinventer l'Occident, chercher dans l'ailleurs méditerranéen les racines de sa propre civilisation}

12 Face aux musulmans, les Européens cherchèrent dans l'autre méditerranéen les racines de leur civilisation ainsi que des moyens de l'améliorer. Longtemps après la pèlerine et mystique anglaise Margery Kempe (v. 1373-après 1438), partie du Norfolk à Jérusalem, des femmes, elles aussi britanniques, occupèrent une place éminente dans l'« apprivoisement » de l'Orient méditerranéen par les Européens à partir du XVIII ${ }^{\mathrm{e}}$ siècle. Ces expériences sont aux antipodes de la représentation des femmes soumises aux hommes. Lady Montagu, épouse de l'ambassadeur anglais à Constantinople, inaugura en 1716 une saison de transferts médicaux avec l'Europe en acceptant de faire inoculer son fils contre la variole puis en demandant, de retour à Londres, l'inoculation pour sa fille. D'autres femmes la suivirent, affirmant en Orient leur autonomie. Lady Craven initia le mouvement en complétant en 1785-1786 son tour d'Europe de Paris à Saint-Pétersbourg par la Crimée, Constantinople, les îles grecques et la Transylvanie. Elle accomplit plus de huit mille kilomètres en quatorze mois et contribua au lancement du genre littéraire du voyage au féminin ${ }^{16}$. Parmi celles qui se mirent en route après elle dans la première moitié du XIXe siècle, on compte autant des Britanniques, comme Julia Pardoe, Emma Roberts, Isabella Romer ou Florence Nightingale, que l'Italienne Amalia Nizzoli, qui rapporta des mémoires de son séjour d'une dizaine d'années en Égypte entre 1819 et 1828.

Les voyages en Méditerranée jouèrent également un rôle moteur dans la naissance de l'archéologie. Les «antiquaires » de l'époque, de Spon et Wheler en 1675-1676 à Paul Lucas, montrèrent le chemin en traitant la Méditerranée comme une réserve d'objets rares et précieux, médailles, monnaies ou inscriptions. Pierre-Augustin Guys fut sensible à la Grèce moderne dans son Voyage littéraire de 1771 et l'abbé Jean-Jacques Barthélemy publia une fiction didactique qui eut un grand succès, le Voyage du jeune Anarchasis en Grèce (1788). Le débat s'anima quand commencèrent à se diffuser les dessins de monuments. Les Anglais James Stuart et Nicholas Revett critiquèrent dans les Antiquities of Athens (1764) les approximations des dessins d'Athènes que Julien-David Le Roy avait fait paraître dans les Ruines des plus beaux monuments de la Grèce (1758) : Le Roy contreattaqua, estimant que ses représentations « affectent plus vivement le spectateur et font passer dans son âme l'admiration dont on est frappé en voyant les monuments mêmes ${ }^{17}$. " Si les sites antiques suscitèrent parfois des descriptions austères comme celle de Lechevalier sur la Troade (1798), ils sous-tendirent surtout la vogue des voyages pittoresques, grands in-folios somptueusement illustrés de gravures commentées qui bénéficiaient de l'argent de mécènes prestigieux et passionnés. Ces riches publications, qui ruinèrent certains de leurs promoteurs, s'appliquèrent successivement à la GrandeGrèce, c'est-à-dire à l'Italie méridionale, avec Saint-Non (1781-1786), au Sud de la France avec Jean-Benjamin de La Borde (1781-1796), à la Sicile avec Jean-Pierre Hoüel (1782-1787) et à la Grèce avec le comte de Choiseul-Gouffier (1782-1824) ${ }^{18}$. Il s'y ajouta la Syrie, le Liban, la Palestine et l'Égypte, dont la campagne de dessins d'abord financée par ChoiseulGouffier entre 1784 et 1786 aboutit à une publication en 1799 de deux des trois volumes prévus, par les soins de La Porte du Theil et du dessinateur Louis-François Cassas ${ }^{19}$. L'on ne doit pas oublier non plus l'Istrie et la Dalmatie, publicisées d'abord par Robert Adam (1764) et ensuite par Cassas dont les vues de 1782 parurent vingt ans plus tard, sous le Consulat, avec un texte de Lavallée (1802), ni l'Espagne promue par Alexandre de Laborde 
(1806-1820 $)^{20}$. Le goût des ruines, annoncé par Guys, s'intensifia chez Volney dans son Voyage en Égypte et en Syrie de 1787, qui devint aussi incontournable dans la constitution d'une mémoire de la Méditerranée archéologique que la Description de l'Arabie de Carsten Niebuhr publiée de 1772 à 1778 après son expédition des années 1760 ou les Lettres sur l'Egypte de Savary publiées en 1785-1786.

L'Égypte avait été décrite dès les années 1720-1750 par Claude Sicard, Benoît de Maillet, Richard Pococke et Frederik Ludvig Norden, même si ce fut l'expédition dirigée par Bonaparte qui donna le coup de pouce décisif aux relevés détaillés des monuments pharaoniques, débouchant sur l'imposante Description de l'Égypte de 1809-1822 en dix volumes de textes et treize volumes de planches. Les rives de la Méditerranée devinrent le cadre privilégié d'un ensemble de découvertes qui firent de l'archéologie une science apte à éclairer les Européens sur les origines de leur propre civilisation. Après les dessins levés par N. Revett et J. Stuart en 1751-1753, les Anglais continuèrent à participer à la redécouverte de la Grèce classique avec les planches d'Edward Dodwell consécutives à ses voyages de 1801 et 1805-1806.

Le voyage érudit préluda à la fabrique des mythes littéraires. La presse périodique se fit dès le $\mathrm{xvII}^{\mathrm{e}}$ siècle l'écho de l'intérêt des lettres françaises pour l'Afrique barbaresque (Turbet-Delof, 1973). À côté des marins, pèlerins et diplomates, l'espace méditerranéen fut parcouru par des marchands à la recherche non seulement de pierres précieuses dont ils faisaient commerce, mais aussi de paysages, d'antiquités et de coutumes qu'ils restituaient à leurs lecteurs. Toute marginale qu'elle fut dans les itinéraires d'apprentissage du Grand Tour, la Méditerranée acquit un statut privilégié comme objet de connaissance dans les relations de voyage. Le processus s'accentua au XVIII siècle, au moment même où un attrait thérapeutique incita les Anglais comme Tobias Smollett à transformer la future Côte d'Azur et celle de Sorrente en lieux de villégiature propices à la guérison de maladies comme la mélancolie (Boyer, 2001). C'était préparer le terrain pour qu'au XIX ${ }^{e}$ siècle la Méditerranée devienne une destination en soi, échappant à sa fonction de simple espace intermédiaire entre l'Europe et l'Orient. Une série d'expéditions scientifiques participèrent de cette construction, en fixant l'image d'un milieu pensé comme spécifiquement méditerranéen : celles d'Égypte entre 1798 et 1801, de Morée entre 1829 et 1831, d'Algérie entre 1832 et 1842 (Bourguet et al., 1998). Après que les écrivains romantiques ou bientôt réalistes eussent contribué à en populariser le désir, de Chateaubriand à Byron et de Lamartine à Nerval, Gautier ou Flaubert, ses rivages se transformèrent pour un public élargi sous la reine Victoria en un berceau de la "passion méditerranéenne " décrite par John Pemble (Pemble, 1987). Par-delà les descriptions encyclopédiques et "statistiques" du XvIII ${ }^{e}$ siècle, les îles et l'espace maritime lui-même pouvaient commencer à devenir dans l'imaginaire des voyageurs européens ou américains l'objet d'un engouement qui allait en promouvoir une dimension mythique, apte à rassembler les morceaux épars des expériences antérieures. 


\section{BIBLIOGRAPHIE}

BonNin Jean-Paul (2001), Les Villes du Grand Voyage : espaces et représentations à partir des récits de pèlerinage à Jérusalem (1480-1700), thèse de doctorat, Université Paris 1 Panthéon Sorbonne. Bourguet Marie-Noëlle, LePetit Bernard, Nordman Daniel \& SinARellis Maroula [dir.] (1998), L'invention scientifique de la Méditerranée : Égypte, Morée, Algérie, Paris : Éditions de l'EHESS. BOYER Marc (2001), L'invention de la Côte d'Azur : l'hiver dans le Midi, La Tour-d'Aigues : Éditions de l'Aube.

BRAHIMI Denise (1976), Voyageurs français du XVIII siècle en Barbarie, Atelier Reproduction des thèses, Université Lille 3 ; Paris : diffusion H. Champion.

BRAUDEL Fernand (1949), La Méditerranée et le monde méditerranéen à l'époque de Philippe II, Paris : Armand Colin.

CORBIN Alain (1988), Le territoire du vide : l'Occident et le désir du rivage (1750-1840), Paris : Aubier. DAKHLIA Jocelyne \& VINCENT Bernard [dir.] (2011), Les musulmans dans l'histoire de l'Europe, Une intégration invisible (t. 1), Passages et contacts en Méditerranée (t. 2), Paris : A. Michel.

DAvis Robert Charles (2006), Esclaves chrétiens, maîtres musulmans. L'esclavage blanc en Méditerranée (1500-1800), Paris : Éditions Jacqueline Chambon.

Delumeau Jean (1978), La Peur en Occident, XIVe-XVIIIe siècle, Paris : Fayard.

GOMEZ-GÉRAUD Marie-Christine (1999), Le crépuscule du grand voyage : les récits des pèlerins à Jérusalem (1458-1612), Paris : Champion.

KAISER Wolfgang [dir.] (2008), Le Commerce des captifs : les intermédiaires dans l'échange et le rachat des prisonniers en Méditerranée, $X V^{e}-X V I I I^{e}$ siècle, Rome : École française de Rome.

MOUREAU François [dir.] (2008), Captifs en Méditerranée (XVIe-XVIII siècle). Histoires, récits et légendes, Paris : PUPS

NORDMAN Daniel (2011), Tempête sur Alger. L'expédition de Charles Quint en 1541, Saint-Denis : Bouchène.

Pelletier Monique \& BergÈs Louis [dir.] (2008), Voyages en Méditerranée de l'Antiquité à nos jours, Paris : CTHS.

Pemble John (1987), The Mediterranean Passion. Victorians and Edwardians in the South, Oxford: Clarendon press.

PouILLon François [dir.] (2008), Dictionnaire des orientalistes de langue française, Paris : Karthala. POUMARÈDE Géraud (2006), « Négociants, voyageurs ou captifs musulmans », M. Arkoun (dir.), Histoire de l'Islam et des musulmans en France du Moyen Âge à nos jours, Paris : A. Michel, 394-405. REQUEMORA Sylvie \& LINON-CHIPON Sophie [dir.] (2002), Les Tyrans de la mer : pirates, corsaires et flibustiers, Paris : PUPS.

RUTA Carlo (2009), Viaggiatori arabi nella Sicilia medievale, Catane : Bibliomedi. 
SHAHADEH Salman (2016), Méthode et moyens de l'écriture de l'histoire des pays de l'Orient méditerranéen (Turquie, Syrie, Terre sainte et Égypte) en France durant les XVII et XVIII ${ }^{e}$ siècles (thèse de doctorat), Université Grenoble-Alpes, Grenoble.

TINGUELY Frédéric (2000), L'écriture du Levant à la Renaissance : enquête sur les voyageurs français dans l'Empire de Soliman le Magnifique, Genève : Droz.

TURBET-Delof Guy (1973), L'Afrique barbaresque dans la littérature française aux XVI et XVII e siècles, Genève : Droz.

VALENSI Lucette (2012), Ces étrangers familiers : musulmans en Europe, $\mathrm{XVI}^{e}$-XVIII ${ }^{e}$ siècles, Paris : Payot.

\section{ANNEXES}

Gilles Bertrand est professeur d'histoire moderne et membre du LUHCIE à l'Université Grenoble-Alpes ainsi que membre senior de l'Institut Universitaire de France. Spécialiste de l'histoire de l'Italie et des voyages en Europe au XVIII ${ }^{e}$ siècle, il fait se croiser l'histoire matérielle des mobilités avec celle des savoirs et des représentations sur le monde. Parmi ses ouvrages : Le Grand Tour revisité: le voyage des Français en Italie, milieu XVIII -début $X I X^{e}$ siècle (2008), Rome : École française de Rome ; Histoire du carnaval de Venise, $\mathrm{XI}^{e}-\mathrm{XXI}{ }^{e}$ siècle (2013), Paris : Pygmalion ; (avec J.-Y. Frétigné et A. Giacone), La France et l'Italie. Histoire de deux nations sceurs de 1660 à nos jours (2016), Paris : Colin. Parmi ses directions d'ouvrages récentes : (avec P. Serna), La République en voyage, 1770-1830 (2013), Rennes : PUR ; (avec J. Ehrard), édition des Voyages de Montesquieu (2012), Paris/Lyon : Classiques Garnier ; (avec A. Guyot), Des « passeurs » entre science, histoire et littérature. Contribution à l'étude de la construction des savoirs (1750-1840) [2011], Grenoble : ELLUG.

\section{NOTES}

1. Thomas Corneille (1708), Dictionnaire universel géographique et historique (vol.2), Paris: Coignard.

2. Jean Pillehote (1606), Pérégrinations du Sieur Jean Palerne Forésien... où est traicté de plusieurs singularités et antiquités remarquées ès provinces d'Égypte, Arabie déserte... avec la manière de vivre des Mores et Turcs et de leur religion..., Lyon, 9-14.

3. Charles de Brosses (1799), Lettres historiques et critiques sur l'Italie (an VII, lettre IV), Paris: Ponthieu (de nombreuses éditions de ces Lettres familières ont suivi).

4. Jean Coppin (1686), Le Bouclier de l'Europe, ou la Guerre sainte... avec une relation de voyages faits dans la Turquie, la Thébaïde et la Barbarie, Lyon : A. Briasson.

5. Edmond Huguet (1967), Dictionnaire de la langue française du seizième siècle (t. 7), Paris : Didier, 519.

6. Ce régiment, appelé aussi des Volontaires de Saxe, fut levé en 1743 par le maréchal de Saxe (1696-1750) pour garder sa résidence et fut également engagé dans la guerre de Succession d'Autriche aux côtés de la France avant de devenir en 1751 un régiment de dragons.

7. Ainsi ceux de Sir Henry Blount vers le Levant en 1634, de George Courthop vers Constantinople en 1637 ou de William Paston en 1638 vers Alexandrie, le Caire et Jérusalem. D'autres projets en revanche, à la même époque, ne se concrétisèrent pas, comme ceux de John Milton vers la Grèce (1639) et de John Evelyn vers le Levant (1645). 
8. Sous-titre de l'ouvrage dans l'édition parue à Marseille, Pierre Mesnier, 1703. Une seconde édition, au sous-titre inchangé, fut imprimée à Marseille en 1709 chez Pierre Mortier, d'autres, plus complètes, suivront en 1805 et 1824.

9. Pierre Belon du Mans (1553), Les observations de plusieurs singularitez et choses memorables trouvées en Grèce, Asie, Judée, Égypte, Arabie et autres pays estranges, rédigées en trois livres, Paris : G. Corrozet.

10. George Sandys (1615), A Relation of a Journey begun An. Dom. 1610: foure bookes containing a description of the Turkish Empire, of Aegypt, of the Holy Land, of the remote parts of Italy and islands adioyning, Londres : W. Barrett.

11. Guillaume-Antoine Olivier (an IX-XII), Voyage dans l'empire othoman, l'Égypte et la Perse, fait par ordre du gouvernement, pendant les six premières années de la République, Paris : H. Agasse.

12. Pérégrinations du Sieur Jean Palerne, ouvrage cité.

13. Nicolas de Nicolay (1567), Les quatre premiers livres des navigations et pérégrinations orientales, Lyon: Guillaume Rouille; id. (1576), Les navigations, pérégrinations et voyages faicts en la Turquie, Anvers : G. Silvius ; Jacques de Villamont (1595), Les voyages du seigneur de Villamont, divisez en trois livres..., Paris : C. de Monstr'oeil et J. Richer.

14. Jean-Baptiste Tavernier (1676), Les six voyages de Jean Baptiste Tavernier... qu'il a fait en Turquie, en Perse et aux Indes, Paris : G. Clouzier; Jean Thévenot (1665), Relation d'un voyage fait au Levant, Paris : Louis Billaine.

15. Paul Lucas (1704), Voyage du sieur Paul Lucas au Levant (2 vol.), Paris: G. Vandive; Deuxième voyage du sieur Paul Lucas dans le Levant, octobre 1704-septembre 1708 [2002], Saint-Étienne: Publications de l'université de Saint-Étienne ; Troisième voyage du sieur Paul Lucas dans le Levant, mai 1714-novembre 1717 [2004], Saint-Étienne : Publications de l'université de Saint-Étienne.

16. Elizabeth Craven (1789), A Journey Through the Crimea to Constantinople etc, Dublin: Chamberlaine et al.

17. Julien-David Le Roy (1767), Observations sur les édifices des anciens peuples (préface), Amsterdam [en fait Paris] : Joseph Merlin, VI.

18. Jean-Claude Richard de Saint-Non (1781-1786), Voyage pittoresque ou Description des royaumes de Naples et de Sicile (4 vol.), Paris: Clousier; Jean-Benjamin de La Borde (1784-1792), Voyage pittoresque de la France (8 vol.), Paris : Lamy ; Jean-Pierre Houël (1782-1787), Voyage pittoresque des isles de Sicile, de Malte et de Lipari (4 vol.), Paris: Impr. de Monsieur; Marie-Gabriel-FlorentAuguste de Choiseul-Gouffier (1782-1822), Voyage pittoresque de la Grèce (3 vol.), Paris : Tilliard.

19. Voyage pittoresque de la Syrie, de la Phoenicie, de la Palaestine et de la Basse Aegypte (2 vol.), textes de Gabriel de La Porte du Theil, Louis Langlès et Jacques-Guillaume Legrand, dessins de LouisFrançois Cassas (1799, an VII), Paris : impr. de la République.

20. Robert Adam (1764), Ruins of the palace of the Emperor Diocletian at Spalatro in Dalmatia, Londres ; Voyage pittoresque et historique de l'Istrie et de la Dalmatie, rédigé d'après l'itinéraire de L.-F. Cassas, par Joseph Lavallée (1802, an x), Paris : Née ; Alexandre de Laborde (1806-1820), Voyage pittoresque et historique de l'Espagne (4. vol.), Paris : impr. de Didot l'aîné.

\section{RÉSUMÉS}

Lieu de passage traditionnel des pèlerinages vers la Terre sainte qui générèrent de nombreux récits jusqu'au XVII ${ }^{e}$ siècle, la Méditerranée resta tout au long de l'époque moderne la source de 
multiples dangers. Mais tout comme elle avait été sillonnée par les géographes arabes au Moyen Âge, elle vit passer entre $\mathrm{XVI}^{\mathrm{e}}$ et $\mathrm{XVIII}^{\mathrm{e}}$ siècles de nombreux voyageurs qui n'eurent de cesse d'écrire leur expérience. Diplomates et marchands, cartographes et naturalistes y collectèrent des connaissances de tous ordres sur le chemin du Levant, de la Barbarie, de la Perse et de l'Inde. À la fin de l'époque des Lumières, tandis que le Grand Tour s'épuisait sur le continent, la Méditerranée se mit à accueillir le désir d'un ailleurs où les Européens recherchaient les racines de leur civilisation. La mer Méditerranée se transforma à la fois en un mythe et en un milieu autonome, qui cessait de n'être qu'un espace intermédiaire entre l'Occident, l'Afrique et l'Orient pour devenir, en tant que Sud, l'enjeu d'une nouvelle passion des voyageurs venus du Nord.

Traditionally a passageway for pilgrims en route for the Holy land, generating travelogues until the $17^{\text {th }}$ century, the Mediterranean remained a dangerous place throughout the modern period. Explored by Arab geographers during the Middle Ages, it was then visited and described by numerous travellers who reported their experience from the $16^{\text {th }}$ to the $18^{\text {th }}$ century. Diplomats, merchants, cartographers, and naturalists collected information and data on their way to the Levant, the Barbary coast, Persia, and India. By the end of the Enlightenment, as the Grand Tour was wearing out on the continent, the Mediterranean became a locus of desire where Europeans went in quest of the origins of their civilisation. The Mediterranean was then turned into both a myth and an autonomous place; instead of being a mere passageway between the West, Africa and the East, the Mediterranean developped among travellers coming from the north a craze for its southern shores.

INDEX

Keywords : Mediterranean, travel, danger, knowledge, desire, civilisation, origins, myth Mots-clés : Méditerranée, voyage, danger, connaissance, désir, civilisation, racines, mythe

\section{AUTEUR}

\section{GILLES BERTRAND}

Professeur à l'Université Grenoble-Alpes (LUHCIE) 